\title{
Essais de l'appareil "Pro-Milk automatique" destiné au dosage en série de la matière azotée du lait
}

\author{
par \\ R. GRAPPIN et R. JEUNET \\ avec la collaboration technique de R. RIGOGNE \\ I.N.R.A., Station Expérimentale Laitière (39) Poligny
}

\section{INTRODUCTION}

Pour les dosages en série de la matière azotée du lait, on utilise généralement la méthode colorimétrique au Noir Amido. Cette méthode a fait l'objet depuis quelques années d'une mécanisation très poussée, en particulier dans les laboratoires des Pays-Bas (Auclair et al., 1970).

Récemment, un constructeur danois, la Société A/S N Foss Electric (Hiller $\varnothing \mathrm{d}$ ) a mis au point un appareil automatique pour le dosage de la matière azotée du lait : le Pro-Milk Automatique (PMA), dont le principe repose sur la méthode au Noir Amido.

Grâce au prêt d'un appareil par le constructeur, nous avons pu réaliser une série d'essais dont nous donnons ici les résultats.

\section{I. - METHODE ET APPAREIL}

\section{a) Principe}

Nous n'exposerons pas le principe de la méthode au Noir Amido qui est actuellement bien connu (Schober et Hetzel, 1956 ; Ashworth et al., 1960 ; Dolby, 1961 ; Posthumus, 1960), pour ne donner ici que les différences essentielles existant entre la technique utilisée en France (Mocquot et al., 1963) et aux Pays-Bas (Posthumus, 1960) et celle utilisée par le PMA. Dans la technique française et hollandaise on utilise la centrifugation comme procédé pour séparer le complexe protéines-colorant du reste de la solution tandis qu'avec le PMA cette séparation est réalisée par filtration. 


\section{b) Réalisation et fonctionnement de l'appareil}

Toutes les phases du dosage sont résumées dans le schéma de la figure 1. Les flacons de lait étant disposés sur un portoir placé sur un rail d'alimentation, l'appareil prélève par échantillon un volume de $4,5 \mathrm{ml}$ de lait dont une partie sert au rinçage de la seringue et de ses canalisations tandis que l'autre partie, soit $0,75 \mathrm{ml}$, est évacuée dans une chambre de réaction. Un volume de $15 \mathrm{ml}$ de solution colorante est ensuite ajouté au lait puis mélangé pendant $3 \mathrm{~s}$ environ par un agitateur tournant à grande vitesse $(12000 \mathrm{t} / \mathrm{mn})$.

A la partie inférieure de la chambre de réaction se trouve le filtre qui est en fibre de verre traitée au silicone. Lorsque le mélan-

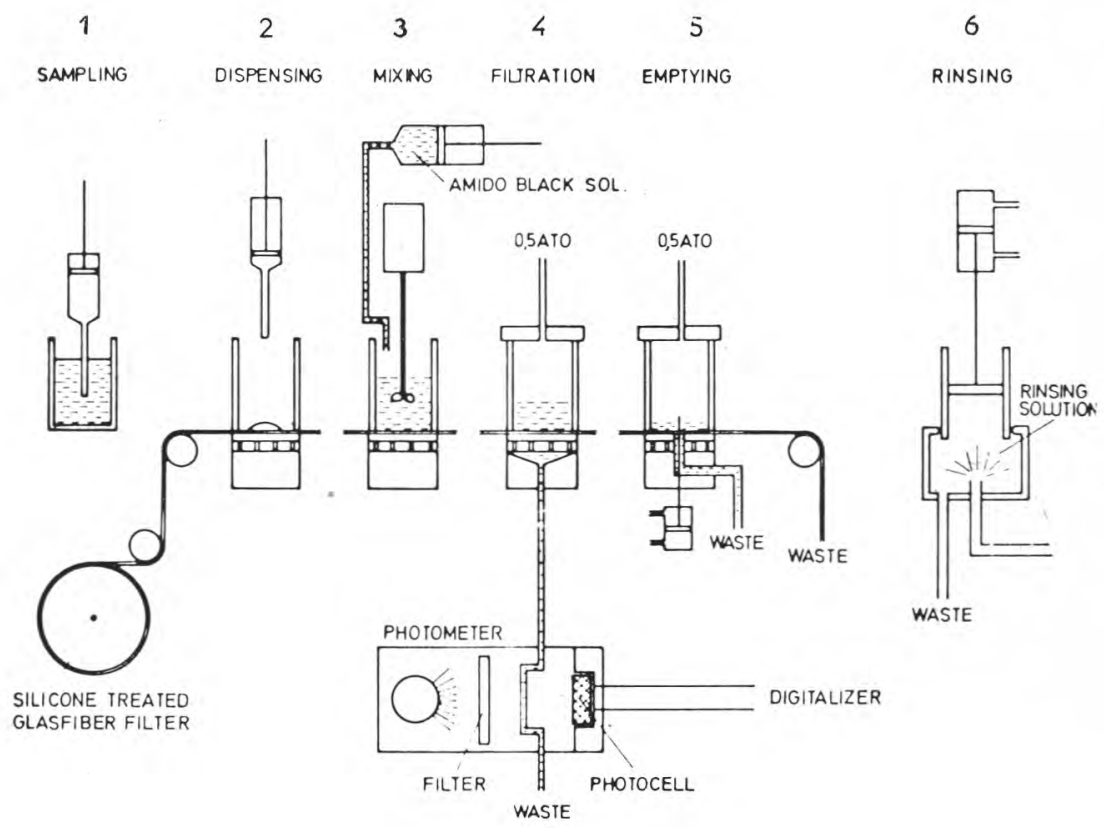

(doc. A/S V Foss Electric)

fig. 1

Principe du Pro-Milk Automatique :

1) Prélèvement du lait.

2) Evacuation du lait dans la chambre de réaction.

3) Addition de la solution de Noir Amido et mélange.

4) Filtration sous pression de 0,5 atmosphère et mesure de la densité optique de la solution.

5) Percement du filtre et évacuation du mélange lait et colorant.

6) Nettoyage de la chambre de réaction. 
ge lait + colorant est terminé, une pression de 0,5 atmosphère est appliquée à la partie supérieure de la chambre entraînant la filtration de la solution de Noir Amido qui est envoyée directement dans une cuve de $0,2 \mathrm{~mm}$ d'épaisseur.

La mesure de la densité optique se fait à une longueur d'onde de $600 \mathrm{~nm}$ et un convertisseur de données permet de lire directement le taux de matière azotée en g p. 1000 . Lorsque la lecture est terminée, le filtre est percé et la solution restante est évacuée. L'appareil est muni de 2 chambres de réaction pivotant autour d'un axe ; pendant qu'une des chambres est utilisée pour le dosage, l'autre est nettoyée avec de l'eau additionnée d'ammoniaque.

Toutes les opérations étant automatiques, l'appareil réalise un dosage toutes les $20 \mathrm{~s}$.

\section{c) Solution colorante}

La composition de la solution préconisée par le constructeur diffère peu de celle qui est utilisée en France et en Hollande (Posthumus, 1960) ; elle comprend par litre de solution : 15,84 g d'acide citrique $\left(\mathrm{C}_{8} \mathrm{H}_{8} \mathrm{O}_{7}, \mathrm{H}_{2} \mathrm{O}\right), 3,98 \mathrm{~g}$ de phosphate disodique $\left(\mathrm{Na}_{2} \mathrm{H} \mathrm{PO}_{4}, 12 \mathrm{H}_{2} \mathrm{O}\right)$ et 900 à $950 \mathrm{mg}$ de Noir Amido en poudre. Le pH de la solution est de 2,50 .

\section{d) Etalonnage de l'appareil}

Bien que l'appareil soit déjà étalonné par le constructeur il est cependant souhaitable, avant toute utilisation, de vérifier et éventuellement de changer l'étalonnage en procédant de la façon suivante : on dose avec précision, par le PMA et par la méthode Kjeldahl, une quarantaine d'échantillons de lait de teneur en matière azotée variable. Suivant les résultats obtenus, on modifie, soit le volume de Noir Amido distribué, soit l'éclairement de la cellule photo-électrique ou le courant de compensation, de façon à obtenir une parfaite concordance entre les résultats PMA et les résultats Kjeldahl. Les réglages de l'étalonnage sont assez simples à réaliser et les vérifications ultérieures se font à l'aide de deux solutions étalons de Noir Amido.

\section{II. - ESSAIS DE L'APPAREIL}

\section{A. - REPETABILITE ET PRECISION}

- Essar 1 : 43 laits individuels dont la teneur en matière azotée varie de 26 à $41 \mathrm{~g} / \mathrm{kg}$ sont dosés avec le PMA, par la méthode Noir Amido classique (avec centrifugation) et par la méthode Kjeldahl (Rowland, 1938). Toutes les déterminations sont faites en double sur des échantillons de lait frais.

- Résultats :

1) Répétabilité : l'écart-type des mesures intra-lait est de 
$0,07 \mathrm{~g} / \mathrm{kg}$ ). Cette valeur est identique à celle qui est obtenue avec la chaîne Noir Amido classique.

2) Précision : entre la méthode de référence Kjeldahl et le PMA nous trouvons un coefficient de corrélation de 0,992 et un écart-type résiduel de $0,48 \mathrm{~g} / \mathrm{kg}$.

\section{B. - INFLUENCE DE LA CONSERVATION DU LAIT}

Dans un premier essai, nous avons déterminé l'influence du produit de conservation, utilisé à des doses et dans des conditions de température de conservation différentes, sur les dosages de matière azotée faits par le PMA mais également et à titre de comparaison, sur les dosages faits par la méthode Noir Amido classique. Ce premier essai ayant permis de fixer les conditions d'emploi des différents produits de conservation, nous avons déterminé, dans un second essai, l'influence du temps de conservation des laits sur les dosages de matière azotée.

\section{a) Influence du produit et des conditions de conservation du lait sur les dosages de matière azotée}

Nous n'avons étudié que les produits de conservation les plus couramment utilisés : le chlorure mercurique pur ou sous la forme de pastille et le dichromate de potassium, utilisé seul ou en mélange avec du sublimé et de l'acide borique.

- Essai 2: Sur 8 échantillons de lait, conservés d'une part avec du sublimé et d'autre part avec du dichromate de potassium, nous avons analysé l'influence des trois facteurs suivants : la dose de produit, la température de conservation et la méthode de dosage. Chaque facteur a été étudié à deux niveaux.

- Dose : dose normale $\left(\mathrm{Hg} \mathrm{Cl}_{2}\right.$ à 0,7 p. 1000 et $\mathrm{K}_{2} \mathrm{Cr}_{2} \mathrm{O}_{7}$ à 1 p. 1000 ) et forte dose ( 5 fois la dose normale).

- Température de conservation : $4^{\circ} \mathrm{C}$ et $20^{\circ} \mathrm{C}$.

- Méthode de dosage : PMA et Noir Amido classique. vation.

Les dosages ont été faits sur le lait frais et après $8 \mathrm{j}$ de conser-

Pour chaque produit de conservation, nous avons fait une analyse de variance sur les résultats des dosages après $8 \mathrm{j}$ de conservation.

- Résultats : Les résultats moyens pour l'ensemble de l'essai sont donnés dans le tableau 1.

1. Influence du sublimé

L'analyse de variance (tab. 2) montre :

1) Une influence hautement significative de la dose de sublimé, qui se traduit par une baisse moyenne de $1,4 \mathrm{~g} / \mathrm{kg}$ du taux de 
TABLEAU 1. - Résultats des dosages de Matière Azotée de l'essai 2

\begin{tabular}{|c|c|c|c|c|c|c|}
\hline \multirow{3}{*}{$\begin{array}{c}\text { Méthode } \\
\text { de } \\
\text { dosage }\end{array}$} & \multirow{3}{*}{$\begin{array}{c}\text { Dose } \\
\text { de } \\
\text { conservateur }\end{array}$} & \multirow{3}{*}{$\begin{array}{c}\text { Température } \\
\text { de } \\
\text { conservation }\end{array}$} & \multicolumn{4}{|c|}{ Produit de conservation } \\
\hline & & & \multicolumn{2}{|c|}{$\mathrm{Hg} \mathrm{Cl}_{2}$} & \multicolumn{2}{|c|}{$\mathrm{K}_{2} \mathrm{Cr}_{2} \mathrm{O}_{7}$} \\
\hline & & & $\bar{x}$ & $\mathrm{~d}$ & $\overline{\bar{x}}$ & $\mathrm{~d}$ \\
\hline Noir Amido & 1 dose & $\begin{array}{r}4^{\circ} \mathrm{C} \\
20^{\circ} \mathrm{C}\end{array}$ & $\begin{array}{l}31,7 \\
31,6\end{array}$ & $\begin{array}{r}+0,2 \\
+0,1\end{array}$ & $\begin{array}{l}33,2 \\
32,9\end{array}$ & $\begin{array}{l}+1,7 \\
+1,4\end{array}$ \\
\hline classique & 5 doses & $\begin{array}{r}4^{\circ} \mathrm{C} \\
20^{\circ} \mathrm{C}\end{array}$ & $\begin{array}{l}31,7 \\
30,3\end{array}$ & $\begin{array}{r}+0,2 \\
-1,2\end{array}$ & $\begin{array}{l}38,8 \\
38,4\end{array}$ & $\begin{array}{l}+7,3 \\
+6,9\end{array}$ \\
\hline \multirow{2}{*}{ PMA } & 1 dose & $\begin{array}{r}4^{\circ} \mathrm{C} \\
20^{\circ} \mathrm{C}\end{array}$ & $\begin{array}{l}31,6 \\
31,5\end{array}$ & $\begin{array}{c}0,1 \\
0\end{array}$ & $\begin{array}{l}31,3 \\
30,8\end{array}$ & $\begin{array}{l}-0,2 \\
-0,7\end{array}$ \\
\hline & 5 doses & $\begin{array}{r}4^{\circ} \mathrm{C} \\
20^{\circ} \mathrm{C}\end{array}$ & $\begin{array}{l}30,1 \\
28,4\end{array}$ & $\begin{array}{l}-1,4 \\
-3,1\end{array}$ & $\begin{array}{l}31,0 \\
30,6\end{array}$ & $\begin{array}{l}-0,5 \\
-0,9\end{array}$ \\
\hline
\end{tabular}

$\mathrm{Hg} \mathrm{Cl}_{2}: 1$ dose $=0,7$ p. $1000 ; \mathrm{Cr}_{2} \mathrm{O}_{7} \mathrm{~K}_{2}: 1$ dose $=1$ p. 1000 .

$\bar{x}$ : moyenne $(\mathrm{g} / \mathrm{kg}) ; \mathrm{d}$ : moyenne des écarts par rapport au lait frais $(\mathrm{g} / \mathrm{kg})$ 
$T A B L E A U 2$

Analyse de variance des résultats des dosages de Matière Azotée dans le cas de laits conservés avec du sublimé (essai 2)

\begin{tabular}{|c|c|c|c|c|}
\hline $\begin{array}{l}\text { Source de } \\
\text { variation }\end{array}$ & $\begin{array}{l}\text { Somme des } \\
\text { carrés }\end{array}$ & $\begin{array}{c}\text { Degré de } \\
\text { liberté }\end{array}$ & $\begin{array}{l}\text { Carré } \\
\text { moyen }\end{array}$ & $\mathrm{F}$ \\
\hline 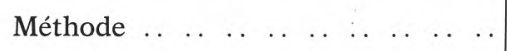 & 13,14 & 1 & 13,14 & $8,02(++)$ \\
\hline 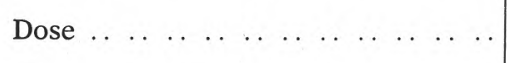 & 33,93 & 1 & 33,93 & $20,70(++)$ \\
\hline 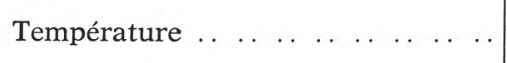 & 10,24 & 1 & 10,24 & $6,25(+)$ \\
\hline Méthode $\times$ dose $\ldots \ldots \ldots c c c c c$ & 10,56 & 1 & 10,56 & $6,44(+)$ \\
\hline Méthode $\times$ température $\ldots \ldots \ldots$.. & 0,076 & 1 & 0,076 & 0,05 (NS) \\
\hline Dose $\times$ température $\ldots \ldots \ldots \ldots$ & 8,27 & 1 & 8,27 & $5,04(+)$ \\
\hline 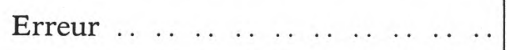 & 93,41 & 57 & 1,639 & \\
\hline $\begin{array}{lllllllllll}\text { Totale } & \ldots & \ldots & \ldots & \ldots & . & \ldots & \ldots & \ldots & \ldots & . .\end{array}$ & 169,63 & 63 & & \\
\hline
\end{tabular}




\section{TABLEAU 3}

Analyse de variance des résultats des dosages de Matière Azotée dans le cas de laits conservés avec du dichromate de potassium (essai 2)

\begin{tabular}{|c|c|c|c|c|}
\hline $\begin{array}{l}\text { Source de } \\
\text { variation }\end{array}$ & $\begin{array}{l}\text { Somme des } \\
\text { carrés }\end{array}$ & $\begin{array}{c}\text { Degré de } \\
\text { liberté }\end{array}$ & $\begin{array}{l}\text { Carré } \\
\text { moyen }\end{array}$ & F \\
\hline 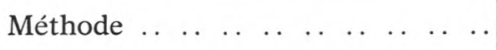 & 385,14 & 1 & 385,14 & $259,35(++)$ \\
\hline Dose $\ldots \ldots \ldots \ldots \ldots \ldots \ldots$ & 112,89 & 1 & 112,89 & $71,22(++)$ \\
\hline Température $\ldots \ldots \ldots \ldots \ldots$ & 2,32 & 1 & 2,32 & 1,46 (NS) \\
\hline Méthode $\times$ dose $\ldots \ldots \ldots \ldots \ldots$ & 135,72 & 1 & 135,72 & $85,63(++)$ \\
\hline Méthode $\times$ température $\ldots \ldots \ldots \ldots$ & 0,04 & 1 & 0,04 & $<1 \quad$ (NS) \\
\hline Dose $\times$ température $\ldots \ldots \ldots c \ldots$ & 0,04 & 1 & 0,04 & $<1 \quad$ (NS) \\
\hline $\begin{array}{lllllllllll}\text { Erreur } & . & \ldots & \ldots & \ldots & . & \ldots & \ldots & \ldots & .\end{array}$ & 90,35 & 57 & 1,585 & \\
\hline 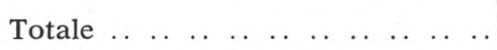 & 726,50 & 63 & & \\
\hline
\end{tabular}


matière azotée lorsque le lait est conservé avec 5 doses au lieu d'une dose.

2) Une influence significative de la méthode de dosage et de la température de conservation : on note une baisse moyenne de $1,0 \mathrm{~g} / \mathrm{kg}$ des dosages PMA par rapport aux dosages Noir Amido classique et de $0,8 \mathrm{~g} / \mathrm{kg}$ des échantillons conservés à $20^{\circ} \mathrm{C}$ par rapport aux échantillons conservés à $4^{\circ} \mathrm{C}$.

3) Des interactions positives entre la méthode d'analyse et la dose de sublimé et entre la dose de sublimé et la température de conservation des laits. Pour l'interaction méthode $x$ dose, l'examen des moyennes indique qu'avec la méthode Noir Amido classique il $\mathrm{y}$ a une baisse moyenne de $0,6 \mathrm{~g} / \mathrm{kg}$ quand on passe de 1 à 5 doses de sublimé ; par contre on constate une baisse plus importante, soit $2,3 \mathrm{~g} / \mathrm{kg}$, avec le PMA quand on augmente la dose. Le PMA est donc plus sensible à l'influence de la concentration en sublimé que le Noir Amido classique. Quant à l'interaction dose $\times$ température, il n'y a pas de différence entre $4^{\circ} \mathrm{C}$ et $20^{\circ} \mathrm{C}$ lorsque les échantillons sont conservés avec une dose de sublimé. Par contre, on observe une baisse moyenne de $1,5 \mathrm{~g} / \mathrm{kg}$ avec 5 doses lorsque les échantillons sont conservés à $20^{\circ} \mathrm{C}$. L'action du sublimé se trouve donc amplifiée si on élève la température de conservation des échantillons. Il en est d'ailleurs de même si on augmente la durée de conservation des laits.

En résumé, l'influence du sublimé sur les dosages de matière azotée par la méthode au Noir Amido est soumise à quatre facteurs principaux : la dose de sublimé, la durée et la température de conservation des laits et la méthode de dosage.

On peut formuler l'hypothèse suivante pour expliquer l'action du sublimé : les protéines du lait réagiraient lentement avec le sublimé au fur et à mesure de l'augmentation de la durée de conservation de cette réaction étant accélérée si la température de conservation est élevée. La "combinaison » formée entre les protéines et le sel de mercure réduirait la capacité de fixation des protéines avec le Noir Amido entraînant ainsi une sous-estimation des résultats des dosages de protéines.

Pour expliquer les différences de résultats entre le PMA et la méthode Noir Amido classique, lorsque les laits sont conservés avec 5 doses de sublimé, on peut supposer que la « combinaison » entre le sublimé et les protéines est détruite, au moins partiellement, par la solution acide de Noir Amido au cours du dosage, si le temps de contact entre le lait et la solution colorante est suffisamment long (comme c'est le cas dans la méthode classique). Nous avons d'ailleurs vérifié que dans le cas d'un lait conservé $2 \mathrm{j}$ à $20^{\circ} \mathrm{C}$ avec 5 doses de sublimé, les résultats ne sont plus sous-estimés si on porte le temps de mélange à $1 / 2 \mathrm{~h}$. Par contre, au-delà de $2 \mathrm{j}$ de conservation, les résultats restent sous-estimés, même après $1 \mathrm{~h}$ de mélange. 


\section{$2^{\circ}$ Influence $d u$ dichromate de potassium}

Le tableau d'analyse de variance (tab. 3) indique une influence hautement significative de la méthode de dosage et de la dose de dichromate. Les dosages faits par la méthode Noir Amido classique sont en moyenne supérieurs de près de $5 \mathrm{~g}$ aux dosages réalisés avec le PMA ; quant aux résultats obtenus avec 1 dose ou 5 doses de dichromate, ils diffèrent de $2,7 \mathrm{~g} / \mathrm{kg}$.

Le dichromate a donc une nette influence sur les dosages par la méthode Noir Amido classique et cette influence semble proportionnelle à sa concentration.

Seule l'interaction méthode de dosage $\times$ dose a été trouvée hautement significative. En effet, si avec le PMA on observe une légère baisse des résultats $(-0,3 \mathrm{~g} / \mathrm{kg}$ en moyenne) quand on augmente la dose de dichromate de 1 à 5 , en revanche les résultats donnés par le Noir Amido classique augmentent très fortement avec la dose de dichromate $(+5,6 \mathrm{~g} / \mathrm{kg}$ avec 5 doses $)$.

Dans l'analyse de variance, l'influence de la température n'a pas été trouvée significative bien que les résultats obtenus sur les laits

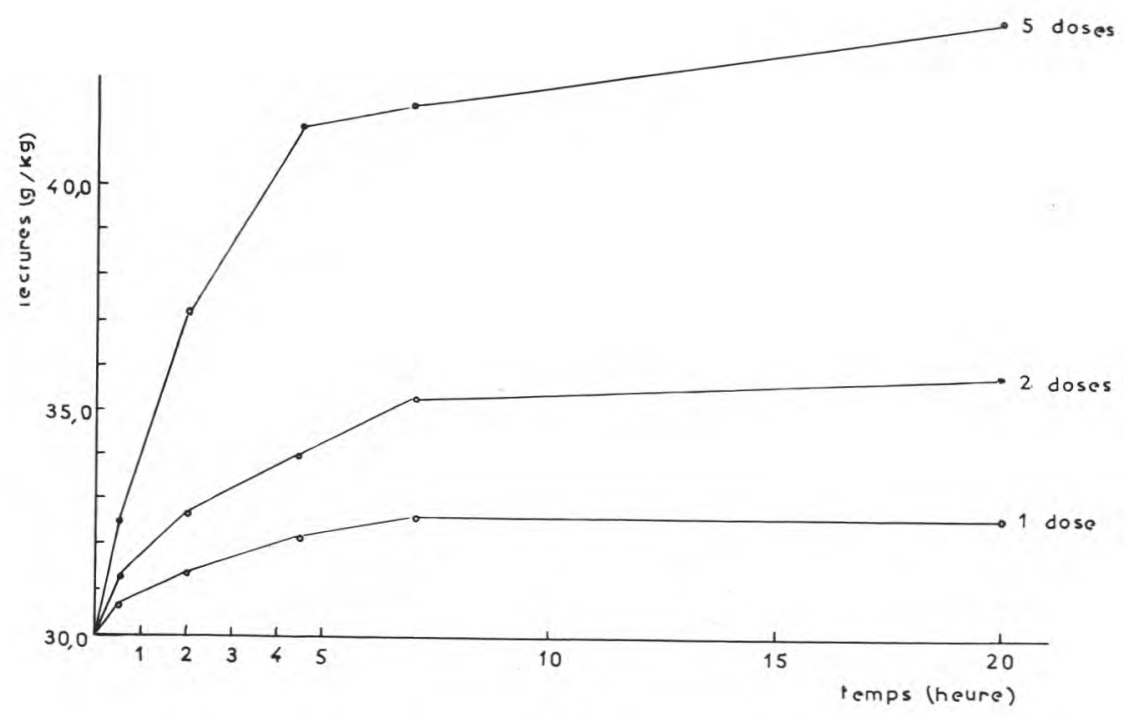

fig. 2

Influence de la concentration du lait en dichromate de potassium ( 1 dose $=1$ p. 1000 ) et du temps d'attente qui sépare le mélange du lait avec la solution colorante de la lecture au colorimètre, sur les résultats des dosages de matière azotée par la méthode au Noir Amido. 
conservés à $20^{\circ} \mathrm{C}$ soient en moyenne plus faibles de $0,4 \mathrm{~g} / \mathrm{kg}$. Par ailleurs, nous avons vérifié que le temps de conservation n'a pas d'influence sur l'action du dichromate.

En définitive, le dichromate n'agirait pas comme le fait le sublimé, pendant la conservation du lait, mais agirait au cours du dosage en augmentant le pouvoir d'absorption de la solution colorante. D'autre part, il semble que cette réaction soit lente puisque la présence du dichromate ne modifie pas les résultats lorsque les dosages sont faits rapidement (cas du PMA). Nous avons vérifié cette hypothèse en déterminant le taux de matière azotée d'un lait additionné de dichromate pour lequel on a fait varier la dose ainsi que le temps de contact avec la solution de Noir Amido surnageante.

La figure 2 indique clairement que les résultats, exprimés en taux de matière azotée, croissent, d'une part avec la dose de dichromate employée et d'autre part avec le temps d'attente qui sépare le mélange du lait avec la solution colorante de la lecture au colorimètre.

D'autres essais ont montré que les observations que nous venons de formuler restent valables si le sublimé ou le dichromate sont employés sous la forme de pastille (avec du $\mathrm{NaCl}$ comme excipient) ou si, à la place du dichromate, on emploie un mélange composé de 5 parties de dichromate, 1 partie de sublimé et 1 partie d'acide borique (à la concentration de 1,4 p. 1000).

\section{b) Influence de l'âge des échantillons}

- Essar 3 : Au cours d'une période de 18 j, nous avons prélevé 8 séries de 30 échantillons de lait chacune. Les laits sont conservés d'une part avec une pastille de sublimé $\left(0,7\right.$ p. 1000 de $\left.\mathrm{Hg} \mathrm{Cl}_{2}\right)$ et d'autre part du mélange bichromaté $\left(\mathrm{Cr}_{2} \mathrm{O}_{7} \mathrm{~K}_{2}\right.$ : 5 parties ; $\mathrm{Hg} \mathrm{Cl}_{2}$ : 1 partie $; \mathrm{H}_{3} \mathrm{BO}_{3}$ : 1 partie à la concentration de 1,4 p. 1000 ) et gardés à $4^{\circ} \mathrm{C}$ et $20^{\circ} \mathrm{C}$.

Les dosages avec le PMA des 8 séries de laits qui sont âgés de 1 à $18 \mathrm{j}$ sont faits le même jour et dans un ordre tel qu'on élimine les éventuelles variations d'étalonnage de l'appareil ; les dosages de référence par la méthode Noir Amido classique sont faits, pour chaque série, sur le lait frais.

- Résultats : Les moyennes et les écarts-types des écarts PMANoir Amido classique montrent qu'il n'y a pratiquement pas d'influence de l'âge des échantillons de lait sur les mesures PMA (tab.4). On peut conserver des échantillons de lait jusqu'à $18 \mathrm{j}$, aussi bien à $4^{\circ} \mathrm{C}$ qu'à $20^{\circ} \mathrm{C}$ à condition toutefois que les échantillons de lait soient, au départ, de bonne qualité bactériologique. Notons cependant sur l'ensemble des échantillons une diminution très faible, mais significative ( $\mathrm{P}<0,01)$, des résultats, lorsque les échantillons sont conservés à $20^{\circ} \mathrm{C}$. Il est donc préférable, par prudence, de conserver les échantillons à une température nettement inférieure à $20^{\circ} \mathrm{C}$ et aussi voisine que possible de $4^{\circ} \mathrm{C}$. 
TABLEAU 4

Influence de l'âge des échantillons sur les dosages PMA en fonction des conditions de conservation (essai 3)

\begin{tabular}{|c|c|c|c|c|c|c|c|c|c|}
\hline \multirow{4}{*}{$\begin{array}{c}\text { Série } \\
n^{\circ}\end{array}$} & \multirow{4}{*}{$\begin{array}{c}\text { Age } \\
\text { des } \\
\begin{array}{c}\text { échantillons } \\
\text { (jour) }\end{array}\end{array}$} & \multicolumn{8}{|c|}{ Conditions de conservation } \\
\hline & & \multicolumn{4}{|c|}{ Pastille de sublimé } & \multicolumn{4}{|c|}{ Mélange dichromate } \\
\hline & & \multicolumn{2}{|c|}{$4^{\circ} \mathrm{C}$} & \multicolumn{2}{|c|}{$20^{\circ} \mathrm{C}$} & \multicolumn{2}{|c|}{$4^{\circ} \mathrm{C}$} & \multicolumn{2}{|c|}{$20^{\circ} \mathrm{C}$} \\
\hline & & $\bar{x}$ & $\sigma$ & $\bar{x}$ & $\sigma$ & $\bar{x}$ & $\sigma$ & $\bar{x}$ & $\sigma$ \\
\hline 1 & 1 & $+0,16$ & 0,13 & $+0,16$ & 0,13 & $+0,19$ & 0,13 & $+0,06$ & 0,18 \\
\hline 2 & 2 & $-0,05$ & 0,11 & $-0,07$ & 0,11 & $-0,18$ & 0,18 & $-0,12$ & 0,16 \\
\hline 3 & 3 & $-0,04$ & 0,10 & $-0,05$ & 0,12 & $-0,03$ & 0,11 & $-0,06$ & 0,12 \\
\hline 4 & 6 & $+0,03$ & 0,10 & 0 & 0,13 & $+0,08$ & 0,17 & 0 & 0,13 \\
\hline 5 & 8 & $-0,16$ & 0,11 & $-0,15$ & 0,11 & $-0,21$ & 0,13 & $-0,22$ & 0,15 \\
\hline 6 & 11 & 0 & 0,14 & 0 & 0,16 & $+0,13$ & 0,15 & $-0,03$ & 0,17 \\
\hline 7 & 15 & $-0,11$ & 0,15 & $-0,16$ & 0,14 & $-0,04$ & 0,15 & $-0,22$ & 0,16 \\
\hline 8 & 18 & $+0,11$ & 0,19 & $+0,09$ & 0,20 & $+0,19$ & 0,21 & $+0,06$ & 0,19 \\
\hline & Moyenne & $-0,007$ & 0,13 & $-0,022$ & 0,14 & $+0,016$ & 0,15 & $-0,066$ & 0,16 \\
\hline
\end{tabular}

$\bar{x}$ : Moyenne des écarts PMA-Noir Amido classique $(\mathrm{g} / \mathrm{kg}) ; \sigma$ : Ecart-type des écarts PMA-Noir Amido classique (g/kg). 


\section{III. - UTILISATION DE L'APPAREIL}

\section{a) Personnel}

Comme pour tous les appareils automatiques d'analyse du lait actuellement sur le marché, l'utilisation du PMA ne nécessite pas de la part de l'opérateur une qualification de haut niveau. Cependant, il est indispensable que le laboratoire qui possède un tel appareil dispose d'un technicien de valeur ayant une très bonne connaissance théorique et technique de l'appareil.

\section{b) Pannes}

Au cours de nos essais, nous n'avons pas eu d'incident de fonctionnement. Mais il faut souligner que l'appareil n'a jamais été utilisé en continu et que le nombre de dosages réalisés (seulement $20000 \mathrm{au}$ total) est insuffisant pour juger de sa robustesse.

\section{c) Coût du dosage}

Nous nous limiterons à donner des éléments nécessaires au calcul du prix de revient de l'analyse :

- cadence de l'appareil : 1100 dosages par journée de 8 h,

— prix de l'appareil (novembre 1969) : H.T. $98800 \mathrm{~F}$,

- personnel : $3 / 4$ à 1 technicien à un salaire net mensuel moyen de 900 à $1100 \mathrm{~F}$ et $1 / 10$ de technicien supérieur à un salaire net mensuel moyen de 1700 à $2200 \mathrm{~F}$,

- produits chimiques + eau distillée + papier filtre : environ $0,06 \mathrm{~F}$ par dosage.

\section{TABLEAU 5}

Comparaison des résultats des dosages de matière azotée faits par les méthodes Kjeldahl, Noir Amido classique (NA) et PMA

Coefficient de corrélation au-dessus de la diagonale

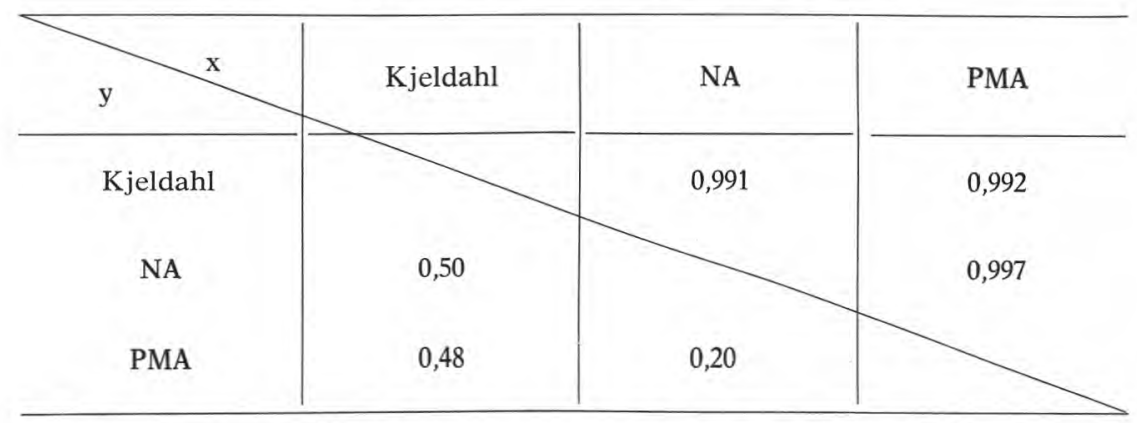

Ecart-type résiduel en dessous de la diagonale $(\mathrm{g} / \mathrm{kg})$ 


\section{DISCUSSION}

Le principe de la méthode au Noir Amido sur laquelle repose le Pro-Milk Automatique ayant déjà fait l'objet de nombreux travaux critiques (voir en particulier l'étude bibliographique de Tarassuk et al., 1967), nous avons limité nos essais à l'étude de la valeur de l'appareil et de ses conditions d'utilisation.

La répétabilité et la précision du PMA sont identiques à celles de la chaîne Noir Amido classique (tab. 5). Nos résultats sont très proches des valeurs annoncées par le constructeur et de celles déjà publiées par O'Keeffe (1968).

Au cours de nos essais, l'étalonnage de l'appareil s'est révélé très stable ; il est cependant recommandé d'utiliser régulièrement un lait de contrôle.

La principale différence entre le PMA et la chaîne classique réside dans l'utilisation de la filtration à la place de la centrifugation. Sur le plan pratique, il s'ensuit qu'on peut réaliser avec le PMA un dosage en $20 \mathrm{~s}$, la filtration étant une opération rapide ( 2 à $3 \mathrm{~s}$ ), tandis qu'avec la chaîne classique utilisant la centrifugation (qui dure $6 \mathrm{mn}$ environ), il faut travailler par série de 90 échantillons ou plus pour arriver à une cadence de dosages du même ordre de grandeur que celle du PMA.

Nous avons vu que l'utilisation des produits de conservation est complexe. Pour résumer, disons qu'on peut utiliser soit le dichromate de potassium à la concentration de 1 p. 1000 , soit le sublimé à la concentration de 0,7 p. 1000 . Ces doses sont également valables dans le cas des échantillons de lait cumulés. Soulignons qu'il faut respecter scrupuleusement les quantités de produit à ajouter au lait car leur influence sur les dosages n'est pas nulle, mais seulement faible aux concentrations envisagées (Curtis et Bennett, 1968).

L'utilisation du PMA présente, pour le laboratoire d'analyse, les avantages et les inconvénients des appareils automatiques. Nous n'aborderons pas ces différents points qui ont déjà été examinés à propos de l'appareil Milko-Tester automatique (Grappin et Jeunet, 1970) avec lequel, rappelons-le, le PMA peut être couplé permettant ainsi le dosage simultané de la matière grasse et de la matière azotée du lait.

\section{R é s u m é}

L'appareil Pro-Milk Automatique (PMA) est basé sur la méthode colorimétrique au Noir Amido. Les propriétés et caractéristiques de l'appareil découlent de cette méthode. Par rapport aux chaînes de dosages employées en France, la différence essentielle réside dans l'utilisation de la filtration à la place de la centrifugation. 
La répétabilité de l'appareil (écart-type intra-lait) est estimée à $0,07 \mathrm{~g} / \mathrm{kg}$ et sa précision (écart-type résiduel) par rapport à la méthode Kjeldahl est de $0,48 \mathrm{~g} / \mathrm{kg}$ pour des laits individuels, avec un coefficient de corrélation de $0,992(n=41)$. Ces valeurs sont identiques à celles qui sont obtenues avec les chaînes classiques utilisant la centrifugation.

Le PMA et la méthode Noir Amido classique réagissent différemment à la conservation du lait avec $\mathrm{Hg} \mathrm{Cl}_{2}$ ou $\mathrm{Cr}_{2} \mathrm{O}_{7} \mathrm{~K}_{2}$. Employé à forte concentration (3,5 p. 1000$)$ le sublimé entraîne une baisse des résultats qui dépend du temps et de la température de conservation du lait ainsi que de la méthode de dosage. Ainsi, pour des laits conservés $8 \mathrm{j}$ à $4^{\circ} \mathrm{C}$ et $20^{\circ} \mathrm{C}$ on obtient respectivement une différence (par rapport aux dosages sur le lait frais) de : $-1,4$ et $-3,1 \mathrm{~g} / \mathrm{kg}$ avec le PMA et $+0,2$ et $-1,2 \mathrm{~g} / \mathrm{kg}$ avec le Noir Amido classique $(\mathrm{n}=8)$. Il semble que le sublimé se " combine » aux protéines du lait au cours de la conservation pour être ensuite déplacé pendant la réaction avec le Noir Amido.

L'addition de $\mathrm{Cr}_{2} \mathrm{O}_{i} \mathrm{~K}_{2}$ au lait provoque une légère baisse des mesures avec le PMA et une très forte augmentation avec le Noir Amido classique. Son action ne dépend pas des conditions de conservation du lait mais du temps de réaction entre le lait et la solution colorante.

Avec le PMA on peut utiliser le sublimé $(0,7$ p. 1000$)$ ou le dichromate de potassium (1 p. 1000). Seul le sublimé peut être employé avec le Noir Amido classique.

Jusqu'à $18 \mathrm{j}$, il n'y a pratiquement pas d'influence de l'âge des échantillons de lait sur les dosages avec le PMA.

Des indications sont données pour le calcul du coût des dosages.

\section{S u m m a ry}

The Pro-Milk Automatic instrument (PMA) is based on the AmidoBlack dye-binding method. Compared with the French Amido-Black procedure, the main difference is the use of filtration instead of centrifugation.

When applied to individual milks, the repeatability of PMA (intra-milk standard deviation) is $\pm 0,007$ p. 100 and compared to the Kjeldahl method, the accuracy expressed as the standard deviation of estimate, is $\pm 0,048$ p. 100 , with $r=0,992(n=41)$. These values are comparable to the results given by the usual Amido-Black procedure (with centrifugation).

The effect of the preservatives, $\mathrm{HgCl}_{2}$ and $\mathrm{K}_{2} \mathrm{Cr}_{2} \mathrm{O}_{7}$ on dyebinding have been studied. A high concentration $(0,35$ p. 100) of mercuric chloride lowers the results : this influence depends upon the temperature, duration of storage and technique of analysis. For exemple, when comparing with results on fresh samples, one gets after 8 days of storage at $4^{\circ} \mathrm{C}$ and $20^{\circ} \mathrm{C}$, respectively : $-0,14$ and 
$-0,31$ p. 100 with PMA and $+0,02$ and $-0,12$ p. 100 with the other method $(\mathrm{n}=8)$.

By adding $\mathrm{K}_{2} \mathrm{Cr}_{2} \mathrm{O}_{7}$ to milk a slight decrease of the readings with PMA is observed and a very high increase with the usual method. The influence of $\mathrm{K}_{2} \mathrm{Cr}_{2} \mathrm{O}_{7}$ is not affected by the storage conditions of milk but by the time during which the milk and the dye-solution react together.

With the PMA, pratically no difference is observed until 18 days of storage at $4^{\circ} \mathrm{C}$ or $20^{\circ} \mathrm{C}$ with normal concentrations of preservatives : $\mathrm{Hg} \mathrm{Cl}_{2}(0,7$ p. 1000$)$ or $\mathrm{K}_{2} \mathrm{Cr}_{2} \mathrm{O}_{7}$ (1 p. 1000$)$. We cannot use $\mathrm{K}_{2} \mathrm{Cr}_{2} \mathrm{O}_{7}$ with the usual method.

Some data are given to calculate the cost of the analysis.

\section{Références bibliographiques}

- Ashworth (U.S), Seals (R.) and ERB (R. E.) (1960). - An improved procedure for the determination of milk protein by dye-binding. J. Dairy Sci., 43, 614-23.

- Auclair (J.), Grappin (R.), Lapied (L.), Muller (M.) et Richard (J.) (1970). Le paiement du lait suivant sa qualité hygiénique et sa composition aux Pays-Bas. Rev. Lait franç., $n^{\circ}$ 278, 511-16.

- Curtis (I. C.) and BennetT (R. A.) (1968). - Effect of mercuric chloride preservative on the Pro-Milk analysis of milk. Aust. J. Dairy Technol., 23, 172-73.

- Dolby (R. M.) (1961). - Dye-binding method for estimation of protein in milk. J. Dairy Res., 28, 43-54.

- Grappin (R.) et Jeunet (R.) (1970). - Essais de l'appareil « Milko-Tester Automatique " destiné au dosage en série de la matière grasse du lait. Le Lait, 50, 233-56.

- Mocouot (G.), Blanc-Patin (E.) et Jeunet (R.) (1962). - La méthode pratique de dosage des protéines du lait. Bull. Techn. d'Inf., 168, 375-82.

- O'KeEfFe (M. G.) (1968). - Automatic Milk fat and protein testing in Scotland. Dairy Inds., 33, 683-84.

- Posthumus (G.) (1960). - Be Bepaling van het eiwitgehalte in melk op grate schaal door middel van de Kleurstofbindingsmethode. Neth. Milk Dairy J., 14, 319-32.

- Rowland (S. J.) (1938). - The determination of the nitrogen distribution in milk. J. Dairy Res., 9, 42-46.

- Schober (R.), Hetzel (H. F.) (1956). - Uber eine einfache kolorimetrische Bestimmung von Milchproteinen. Milchwissenschaft, 11, 123-26.

- TARAssuk (N.P.), ABE (N.) and RoATs (W. A.) (1967). - The dye-binding of milk proteins. Thechn. Bull., no 1369. Agricultural Research service. U.S. Dep. of Agriculture.

Reçu pour publication le 6 octobre 1970.

\section{Remerciements}

Nous remercions vivement $M$. Mocquot pour l'intérêt qu'il a manifesté envers notre travail et pour les corrections apportées au manuscrit. 